\title{
Linear Hyperfine Tuning of Donor Spins in Silicon Using Hydrostatic Strain
}

\author{
J. Mansir, ${ }^{1}$ P. Conti, ${ }^{1}$ Z. Zeng, ${ }^{2}$ J. J. Pla, ${ }^{3}$ P. Bertet ${ }^{4}$ M. W. Swift, ${ }^{5}$ C. G. Van de Walle, ${ }^{5}$ M. L. W. Thewalt, ${ }^{6}$ \\ B. Sklenard, ${ }^{7}$ Y. M. Niquet, ${ }^{2}$ and J. J. L. Morton ${ }^{1,8}$ \\ ${ }^{1}$ London Centre for Nanotechnology, UCL, 17-19 Gordon St, London WC1H OAH, United Kingdom \\ ${ }^{2}$ Université Grenoble Alpes, CEA, INAC-MEM, L_Sim, F-38000 Grenoble, France \\ ${ }^{3}$ School of Electrical Engineering \& Telecommunications, University of New South Wales, Sydney, NSW 2052, Australia \\ ${ }^{4}$ Quantronics Group, SPEC, CEA, CNRS, Université Paris-Saclay, CEA-Saclay, 91191 Gif-sur-Yvette, France \\ ${ }^{5}$ Materials Department, University of California, Santa Barbara, California 93106-5050, USA \\ ${ }^{6}$ Department of Physics, Simon Fraser University, Burnaby, British Columbia V5A 1S6, Canada \\ ${ }^{7}$ Université Grenoble Alpes \& CEA, LETI, MINATEC Campus, F-38000 Grenoble, France \\ ${ }^{8}$ Dept of Electronic and Electrical Engineering, UCL, London WC1E 7JE, United Kingdom
}

(Received 29 September 2017; published 20 April 2018)

\begin{abstract}
We experimentally study the coupling of group $\mathrm{V}$ donor spins in silicon to mechanical strain, and measure strain-induced frequency shifts that are linear in strain, in contrast to the quadratic dependence predicted by the valley repopulation model (VRM), and therefore orders of magnitude greater than that predicted by the VRM for small strains $|\varepsilon|<10^{-5}$. Through both tight-binding and first principles calculations we find that these shifts arise from a linear tuning of the donor hyperfine interaction term by the hydrostatic component of strain and achieve semiquantitative agreement with the experimental values. Our results provide a framework for making quantitative predictions of donor spins in silicon nanostructures, such as those being used to develop silicon-based quantum processors and memories. The strong spin-strain coupling we measure (up to $150 \mathrm{GHz}$ per strain, for $\mathrm{Bi}$ donors in $\mathrm{Si}$ ) offers a method for donor spin tuning - shifting Bi donor electron spins by over a linewidth with a hydrostatic strain of order $10^{-6}$ - as well as opportunities for coupling to mechanical resonators.
\end{abstract}

DOI: 10.1103/PhysRevLett.120.167701

Donors in silicon present an attractive spin qubit platform, offering amongst the longest coherence times in the solid state $[1,2]$ and single-qubit control with fault-tolerant fidelity $[3,4]$. As with the conventional semiconductor industry, the majority of efforts in donor-based spin qubits are focused on ${ }^{31} \mathrm{P}$ donors [5-12]. The heavier group $\mathrm{V}$ donors ${ }^{75} \mathrm{As},{ }^{121} \mathrm{Sb}$, and ${ }^{209} \mathrm{Bi}$ have recently received substantial interest [13-19], offering larger nuclear spins (up to $I=9 / 2$ for ${ }^{209} \mathrm{Bi}$ ) and correspondingly richer Hilbert spaces that enable up to four logical qubits to be represented in a single dopant atom. Furthermore, "atomic clock transitions" have been identified in ${ }^{209} \mathrm{Bi}$ where spin resonance transition frequencies become first-order insensitive to magnetic field noise, resulting in coherence times of up to $3 \mathrm{sec}$ in ${ }^{28} \mathrm{Si}$ [20].

The exploitation of donor spins in silicon as qubits typically requires their incorporation into nano- and microelectronic devices. This has been used to demonstrate the single-shot read-out of a single ${ }^{31} \mathrm{P}$ donor spin using a

Published by the American Physical Society under the terms of the Creative Commons Attribution 4.0 International license. Further distribution of this work must maintain attribution to the author(s) and the published article's title, journal citation, and DOI. tunnel-coupled silicon single-electron transistor [21,22], and to create hybrid devices in which donor spins are coupled to superconducting resonators [23-25] to develop interfaces between microwave photons and solid-state spins. In both cases, the use of metal-oxide-semiconductor (MOS) nanostructures [26], or patterned superconducting films on silicon [27], involves a combination of materials with coefficients of thermal expansion that differ by up to an order of magnitude [28-32]. The presence of strain in the silicon environment around the donor spin is therefore pervasive when studying such nanodevices at cryogenic temperatures. Furthermore, factors such as optimizing spin-resonator coupling or spinread-out speed motivate the placement of donors close to features such as single-electron transistors [33] or resonator inductor wires [34] where strain is maximal.

Strain modifies the band structure of silicon [35,36], and it has been shown, for example, to contribute to the confinement of single electrons in silicon under nanoscale aluminium gates $[37,38]$. The donor electron wave function is also modified by strain: following the valley repopulation model (VRM) developed by Wilson and Feher [39] within the framework of effective mass theory, an applied uniaxial strain lifts the degeneracy of the six silicon valleys leading to a mixture of the donor ground state $1 s\left(A_{1}\right)$ with the first excited state $1 s(E)$. In this excited state, the hyperfine 
coupling between the donor electron and nuclear spin is zero; therefore, the VRM predicts a quadratic reduction in $A$ as a function of uniaxial strain, as well as a strain-induced anisotropic contribution to the electron $g$ factor. Straininduced perturbations of the donor hyperfine coupling have been observed for P-donor spins in ${ }^{28} \mathrm{Si}$ epilayers, grown on SiGe to yield built-in strains of order $10^{-3}$ [40]piezoelectric contacts on such material have been used to modulate this built-in strain to shift the electron spin resonance frequency by up to $\sim 400 \mathrm{kHz}$ [41].

In this Letter, we report the observation of a straininduced shift in the hyperfine coupling of group $\mathrm{V}$ donors in silicon that is linear (rather than quadratic), and therefore orders of magnitude greater than that predicted by the valley repopulation model of Wilson and Feher [39] for small strains $\left(|\varepsilon| \lesssim 10^{-5}\right)$. We present experimental studies showing strain tuning of the coherent evolution of each of the group $\mathrm{V}$ donor spins $\left({ }^{31} \mathrm{P},{ }^{75} \mathrm{As},{ }^{121} \mathrm{Sb}\right.$, and $\left.{ }^{209} \mathrm{Bi}\right)$, extracting the strain-induced shifts of the hyperfine coupling and electron spin $g$ factor for each, and corroborate the results with a combination of both tight binding and density functional theory calculations that reveal the crucial role of hydrostatic strain in this novel mechanism [42]. In addition to providing essential insights for the use of donor spins in nano- and micronscale quantum devices, our results provide a method for linear tuning of the donor hyperfine interaction with coupling strengths of up to $150 \mathrm{GHz} /$ strain for ${ }^{209} \mathrm{Bi}$ donor spins.

The spin Hamiltonian for a group $\mathrm{V}$ donor in the presence of an external magnetic field $\mathbf{B}=B_{0} \mathbf{z}$ is

$$
\hat{\mathcal{H}}=\left(g_{e} \mu_{B} \hat{\mathbf{S}}_{\mathbf{z}}-g_{n} \mu_{N} \hat{\mathbf{I}}_{\mathbf{z}}\right) B_{0}+A \hat{\mathbf{S}} \cdot \hat{\mathbf{I}},
$$

where $g_{e}$ and $g_{n}$ are, respectively, the electronic and nuclear $g$ factors, $\mu_{B}$ and $\mu_{N}$ are the Bohr and nuclear magnetons, and $\hat{\mathbf{S}}$ and $\hat{\mathbf{I}}$ are the electronic and nuclear spin operators. The Fermi contact hyperfine interaction strength, $A=$ $1.4754 \mathrm{GHz}$ in $\mathrm{Si}: \mathrm{Bi}$, can be expressed as

$$
A=\frac{8 \pi}{3} g_{0} \mu_{B} g_{n} \mu_{N}|\psi(0)|^{2},
$$

where $\psi(0)$ represents the amplitude of the electronic wave function at the nucleus and $g_{0}=2.0023$ is the free electron $g$ factor. The eigenstates of this Hamiltonian describe a Hilbert space of dimension $(2 S+1)(2 I+1)$ with $S=1 / 2$ and $I$ determined by the nuclear spin species, illustrated for the case of ${ }^{209} \mathrm{Bi}: \mathrm{Si}$ in Fig. 1(a). Transitions between these eigenstates obeying the selection rule $\left(\Delta m_{S}, \Delta m_{I}\right)=$ $( \pm 1,0)$ in the high field limit can be driven and detected using pulsed electron spin resonance (ESR) [43].

We use samples of isotopically enriched ${ }^{28} \mathrm{Si}$ doped with $\mathrm{Bi}, \mathrm{Sb}, \mathrm{As}$, and P (see Ref. [44] for more details), mounted with crystal orientation shown in Fig. 1(b). The sample is situated inside a dielectric microwave ESR resonator in an

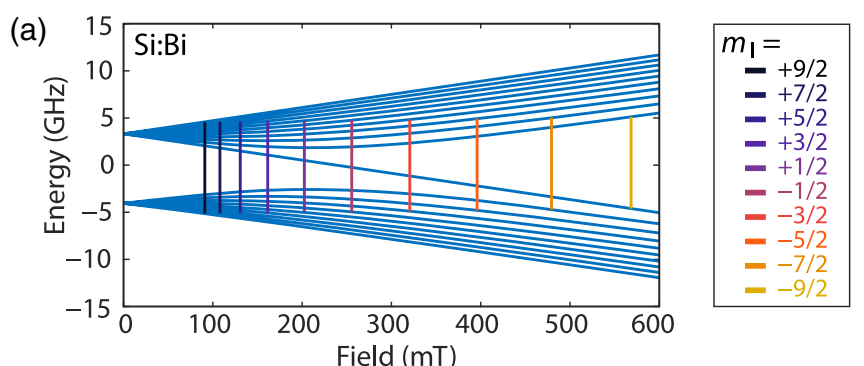

(b)

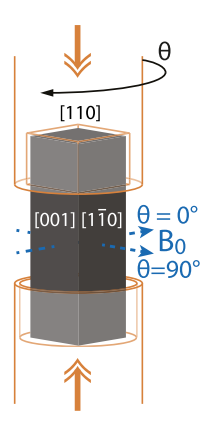

(c)

Strain $\varepsilon_{\mathrm{hs}} \times 10^{-6}$

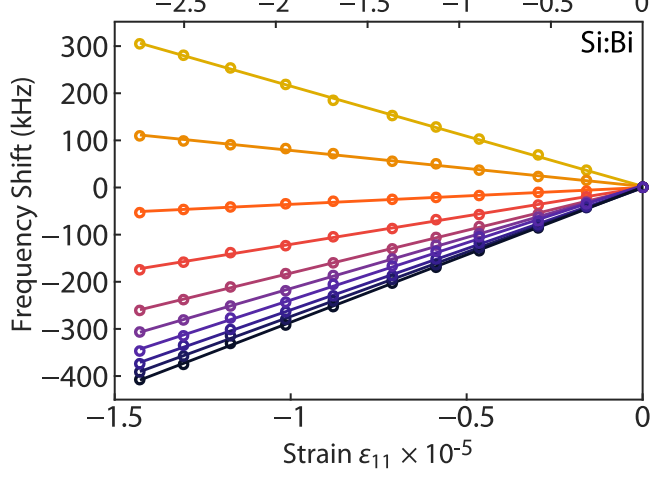

FIG. 1. (a) Energy levels of Si:Bi spin eigenstates as a function of magnetic field, with the ten allowed ESR transitions at $9.7 \mathrm{GHz}$ highlighted and labeled according to their high-field nuclear spin projection $m_{I}$. (b) Schematic of experimental setup. The silicon single crystal sample is mounted between two engineered plastic rods with the ability to apply compressive stress. $\theta$, the angle of the applied magnetic field to the [001] direction, can be varied by rotating the sample. (c) Observed linear frequency shifts for each of the ten allowed ESR transitions shown in panel (a), as a function of strain $\varepsilon_{11}$, with $\theta=30^{\circ}$.

Oxford Instruments CF935 liquid helium flow cryostat, and is held between two plastic (polyether ether ketone) rods whose end faces have been milled using computer numerical control to match the profile of the sample allowing it to be rotated with respect to the magnetic field. Using calibrated masses, a uniaxial stress is applied to the sample perpendicular to the [110] face, via the upper rod, which extends outside the cryostat. The resulting strain tensor can be derived from the generalized form of Hooke's law for anisotropic materials and the compliance matrix for silicon [65]: in the ([110], [110], [001]) coordinate system per kilogram of applied mass, $\varepsilon_{11}=-1.45 \times 10^{-5} / \mathrm{kg}$, $\varepsilon_{22}=9.02 \times 10^{-7} / \mathrm{kg}, \varepsilon_{33}=5.24 \times 10^{-6} / \mathrm{kg}$, and $\varepsilon_{i \neq j}=0$. While the VRM predicts frequency shifts only from uniaxial strain, we shall see that the new mechanism presented here arises from hydrostatic strain $\varepsilon_{\mathrm{hs}}=$ $\left(\varepsilon_{11}+\varepsilon_{22}+\varepsilon_{33}\right) / 3$. In our setup, we estimate a strain per unit mass of $\varepsilon_{\mathrm{hs}}=-2.78 \times 10^{-6} / \mathrm{kg}$.

We use a home-built pulsed ESR spectrometer [66] at 9.7 GHz to apply a Hahn echo sequence $\pi / 2 \rightarrow \tau \rightarrow \pi \rightarrow$ $\tau \rightarrow$ echo [67] with $\tau=15 \mu$ s and a $\pi$ pulse duration of 130 ns. The time-domain Hahn echo signals (top of Fig. 2) obtained while systematically increasing the applied strain 


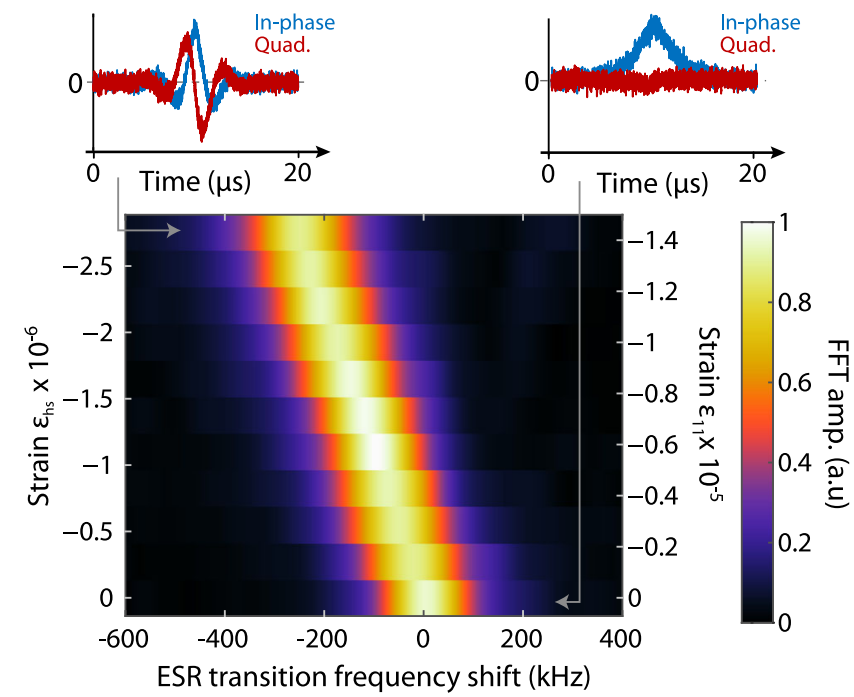

FIG. 2. Electron spin echo signals in the frequency domain measured in ${ }^{28} \mathrm{Si}: \mathrm{Bi}$ as a function of compressive strain (shown in terms of the uniaxial strain $\varepsilon_{11}$ and hydrostatic strain $\varepsilon_{\mathrm{hs}}$ ) arising from the applied stress in our experiment. Time-domain echoes for zero strain and $\varepsilon_{11}=1.4 \times 10^{-5}$ are shown as insets. Data shown are from the $m_{I}=-1 / 2$ transition with $\theta=45^{\circ}$, taken at $T=8 \mathrm{~K}$.

are then Fourier transformed to yield the strain-induced shifts in spin resonance frequency [44].

First, we observe in Fig. 2 that the Bi donor ESR transition can be shifted by more than a linewidth (in ${ }^{28} \mathrm{Si}$ ) for strains of order $10^{-5}$ (uniaxial) or $10^{-6}$ (hydrostatic). We fit the frequency-domain echo signals to a Voigt profile, and then plot the center-frequency shifts as a function of strain, for each of the ten allowed ESR transitions [see Fig. 1(c)]. Strikingly, the ESR frequency of each transition shows a linear dependence on strain, rather than the expected quadratic dependence. In Fig. 3, we plot the experimentally determined $\partial f / \partial \epsilon_{11}$ for each transition against the first-order sensitivity of each transition frequency to the isotropic hyperfine coupling $\partial f / \partial A$. Remarkably, all ten points fall on a single line, demonstrating that the dominant effect we observe in $\mathrm{Si}$ :Bi is a strain-induced shift in the isotropic hyperfine coupling that is linear in strain, and equivalent to $\partial A / \partial \varepsilon_{11}=5.4 \pm$ $0.3 \mathrm{GHz}$ or $\partial A / \partial \varepsilon_{\mathrm{hs}}=28.2 \pm 1.6 \mathrm{GHz}$.

Multivalley effective mass theory has been successful in describing many aspects of the donor electron wave function [68-70], including close agreement between theory and experimental measurements of the Stark effect [71] and predictions of exchange coupling between neighboring donors [72]. Within this framework, the wave function is expanded in terms of Bloch functions concentrated around the six degenerate [100] conduction band minima (valleys) such that $\psi=\sum_{\mu=1}^{6} \alpha_{\mu} F_{\mu} \phi_{\mu}$, where $\mu$ indexes over the valleys in the basis $[+x,-x,+y,-y$, $+z,-z], F_{\mu}$ is a hydrogenlike envelope function, and $\phi_{\mu}$
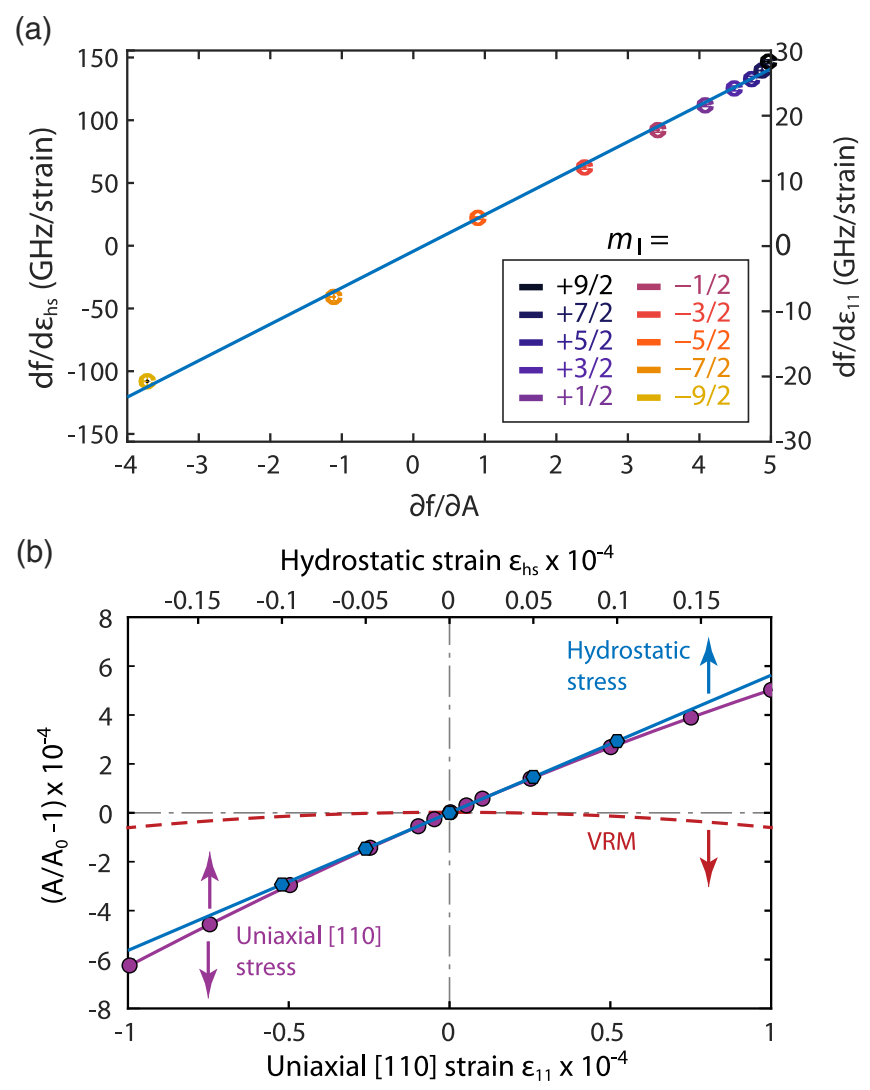

FIG. 3. (a) The gradient of the strain-induced frequency shifts $(d f / d \epsilon)$ for each of the ten Si:Bi ESR transitions are shown as a function of the first-order sensitivity of each transition to the hyperfine coupling $(\partial f / \partial A)$. The linear relationship confirms the observed strain-induced shifts in $\mathrm{Si}: \mathrm{Bi}$ result from tuning the hyperfine coupling, $A$, with a gradient $\partial A / \partial \epsilon_{11}=5.4 \pm 0.3 \mathrm{GHz}$ or, equivalently, $\partial A / \partial \epsilon_{\mathrm{hs}}=28.2 \pm 1.6 \mathrm{GHz}$. (b) Calculations showing the relative change in hyperfine coupling strength $A / A_{0}$ as a function of strain, comparing TB and valley repopulation models. To mimic the experiment, we show TB calculations of $A / A_{0}$ under uniaxial stress along [110] (purple curve and circles), which produces a hydrostatic component of strain ( $\varepsilon_{\mathrm{hs}}$, top axis) in addition to a uniaxial component along [110] $\left(\varepsilon_{11}\right.$, bottom axis). This behavior can be understood by comparing with TB calculations for pure hydrostatic stress (blue curve and hexagons), plotted on the same axis of $\varepsilon_{\mathrm{hs}}$, as well as calculations from the VRM (red dotted curve), plotted on the same axis of $\varepsilon_{11}$. The arrows indicate the relevant axes for each trace.

is the valley Bloch function. The donor impurity potential breaks the symmetry of the crystal and induces a coupling between the valleys, leading to a valley-orbit splitting of the $1 s$-like donor state into three sublevels. The ground state is singly degenerate with $A_{1}$ symmetry and has $\alpha_{\mu}=1 / \sqrt{6}$, while one of the excited states is doubly degenerate with $E$ symmetry and has $\alpha_{E_{1}}=1 / 2[1,1,-1,-1,0,0]$ and $\alpha_{E_{2}}=1 / 2[1,1,0,0,-1,-1]$. The valley repopulation model assumes that uniaxial strain applied along a valley axis results in the corresponding pair of valley energies being decreased or increased for compressive or tensile 
strain, respectively [39]. This modification of the valley energies results in a redistribution of the amplitude of each valley contributing to the ground state, which can be represented under strain as an admixture of the $1 s\left(A_{1}\right)$ and $1 s(E)$ states, resulting in a quadratic reduction of $A$ as a function of uniaxial strain. At our maximum applied strain of $\varepsilon_{11}=-1.45 \times 10^{-5}$, the VRM predicts a reduction in $A$ of $1.9 \mathrm{kHz}$, while we measure a reduction in $A$ of $78 \mathrm{kHz}-$ this discrepancy is even more pronounced for smaller strains. Therefore, in addition to predicting a different functional form of the dependence of $A$ against strain, the VRM predicts shifts that are approximately 2 orders of magnitude smaller than what we measure in this strain regime, implying that another physical mechanism must dominate the changes to the structure of the donor electron wave function we observe.

In order to understand these trends, we have computed the bound states of bismuth impurities in silicon using the $s p^{3} d^{5} s^{*}$ tight-binding (TB) model of Ref. [45]. This model reproduces the variations of the band structure of bulk silicon under arbitrary strains in the whole first Brillouin zone. The impurity is described by a Coulomb tail and by a correction of the orbital energies of the bismuth atom (similar to a central cell correction in the effective mass approximation) [46]. The TB ratio $A / A_{0}$ between the strained $(A)$ and unstrained $\left(A_{0}\right)$ hyperfine interaction strengths is plotted in Fig. 3(b) under uniaxial stress along [110], as a function of the resulting uniaxial [110] and hydrostatic strains. Surprisingly, and in agreement with the experiments performed here, $A / A_{0}$ behaves linearly with small strain, and this trend can be assigned to the effects of the hydrostatic stress. Although not predicted by the VRM, the existence of a linear hydrostatic term is compatible with the symmetries of the system [42]. A symmetry analysis indeed suggests that, to second order in the strains $\varepsilon_{i j}$ in the cubic axis set,

$$
\begin{aligned}
A / A_{0}= & 1+\frac{K}{3}\left(\varepsilon_{x x}+\varepsilon_{y y}+\varepsilon_{z z}\right) \\
& +\frac{L}{2}\left[\left(\varepsilon_{y y}-\varepsilon_{z z}\right)^{2}+\left(\varepsilon_{x x}-\varepsilon_{z z}\right)^{2}+\left(\varepsilon_{x x}-\varepsilon_{y y}\right)^{2}\right] \\
& +N\left(\varepsilon_{y z}^{2}+\varepsilon_{x z}^{2}+\varepsilon_{x y}^{2}\right) .
\end{aligned}
$$

A fit to the TB data yields $K=29.3, L=-9064$, and $N=-225$. $L$ mostly results from the coupling of the $1 s\left(A_{1}\right)$ with the $1 s(E)$ state by the uniaxial strain. The TB $L$ is close to the VRM $L=-2 \Xi_{u}^{2} /\left(9 \Delta^{2}\right)=-9720$ [39], where $\Xi_{u}=8.6 \mathrm{eV}$ is the uniaxial deformation potential of the conduction band of silicon and $\Delta=41 \mathrm{meV}$ is the splitting between the $1 s\left(A_{1}\right)$ and the $1 s(E)$ state of the $\mathrm{Bi}$ impurity. The quadratic shear term $N$ is usually negligible with respect to $L$. $K=\partial\left(A / A_{0}\right) / \partial \varepsilon_{\mathrm{hs}}$ results from the coupling of the $1 s\left(A_{1}\right)$ with the $2 s\left(A_{1}\right)$ state (and higher $A_{1}$ states, since hydrostatic strain preserves the symmetry of the system) due to the change of the shape and depth of the central cell correction under strain. $A / A_{0}$ is dominated by this hydrostatic term at small strain, as evidenced in Fig. 3(b). The TB $K=29.3$ is larger than the experimental $K=19.1$. At variance with $L$ (which mostly depends on a deformation potential of the silicon matrix), $K$ indeed depends on details of the potential near the impurity, which must be specifically accounted for in the TB model in order to reach quantitative accuracy [44]. In order to better capture the central cell correction around the bismuth impurity, we also performed first principles calculations using density functional theory (DFT) to describe the atomic relaxations not accounted for by our TB calculations [44]. The DFT calculations further corroborate the linear dependence of the hyperfine coupling on hydrostatic strain (for $\epsilon_{\mathrm{hs}} \leq 10^{-3}$ ), and predict a coefficient $K=17.5$, in good agreement with our experiments. Full details concerning the models and calculations can be found in Ref. [44].

To test our model further and explore the expected anisotropy of a $g$ factor coupling to strain, we extend our study over a range of magnetic field orientations [as defined in Fig. 1(b)] and for the other group V donors: ${ }^{31} \mathrm{P},{ }^{75} \mathrm{As}$, and ${ }^{121} \mathrm{Sb}$. In all cases we find the observed ESR transition frequency shifts $f$ are linear as a function of hydrostatic strain $\epsilon_{\mathrm{hs}}$, with the resulting coupling strengths $\left(d f / d \epsilon_{\mathrm{hs}}\right)$ summarized in Fig. 4 and Table SI, along with values predicted from tight-binding calculations and full data sets [44]. While we find no significant anisotropy in $\mathrm{Si}$ : $\mathrm{Bi}$, the data from $\mathrm{Si}: \mathrm{Sb}, \mathrm{Si}: \mathrm{As}$, and $\mathrm{Si}: \mathrm{P}$ display strain effects that clearly depend on the magnetic field orientation, attributed to a strain-induced anisotropic electronic $g$ factor. Following Wilson and Feher [39], our model for this anisotropy includes a term accounting for the effect of valley repopulation, and another accounting for the effect

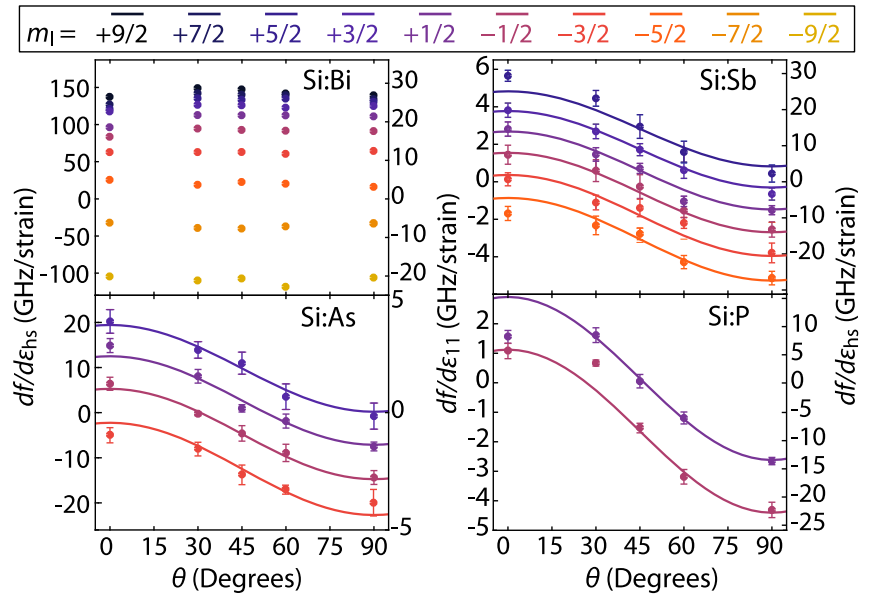

FIG. 4. Extracted linear fit gradients $d f / d \epsilon_{11}$ for each transition for all four donors under consideration as a function of the angle of $B_{0}$ with respect to the crystal $\theta$. For $\mathrm{Si}: \mathrm{Sb}, \mathrm{Si}: \mathrm{As}$, and $\mathrm{Si}: \mathrm{P}$, these fits are overlaid with a model taking into account the linear shift of hyperfine interaction strength $A$ as well as an anisotropic $g$ factor as a function of $\epsilon_{11}$. 
of spin-orbit coupling in the sheared lattice. Fits of this model to our experimental data reproduce the predicted strength of both of these effects to within a factor of 2 [44].

Through experiments and calculations, we have demonstrated that hydrostatic strain in silicon leads to a strong, linear tuning of the hyperfine interaction in group $\mathrm{V}$ donors, through coupling between the $1 s\left(A_{1}\right)$ and $2 s\left(A_{1}\right)$ states. The ability to shift the ESR transition frequencies by over a linewidth with hydrostatic strain in the order of $10^{-6}$ opens up new possibilities for conditional "A-gate" control of donors as well as coupling to mechanical resonators. In addition, these insights will be crucial in supporting the design of quantum memories and processors based on donors in silicon, enabling the ability to accurately predict ESR transition energies as a function of donor position within the device structure.

We acknowledge helpful discussions with Ania Jayich. This research was supported by the Engineering and Physical Sciences Research Council (EPSRC) through UNDEDD (EP/K025945/1) and a Doctoral Training Grant, as well as by the European Union's Horizon 2020 research and innovation programme under Grant Agreement No. 688539 (MOS-QUITO) and the European Community's Seventh Framework Programme No. 279781 (ASCENT) and No. 615767 (CIRQUSS); and also by the Agence Nationale de la Recherche through project QIPSE.

[1] A. M. Tyryshkin, S. Tojo, J. J. L. Morton, H. Riemann, N. V. Abrosimov, P. Becker, H.-J. Pohl, T. Schenkel, M. L. W. Thewalt, and K. M. Itoh, Nat. Mater. 11, 143 (2011).

[2] K. Saeedi, S. Simmons, J. Z. Salvail, P. Dluhy, H. Riemann, N. V. Abrosimov, P. Becker, H.-J. Pohl, J. J. L. Morton, and M. L. W. Thewalt, Science 342, 830 (2013).

[3] J. J. Pla, K. Y. Tan, J. P. Dehollain, W. H. Lim, J. J. L. Morton, F. A. Zwanenburg, D. N. Jamieson, A. S. Dzurak, and A. Morello, Nature (London) 496, 334 (2013).

[4] J. T. Muhonen, A. Laucht, S. Simmons, J. P. Dehollain, R. Kalra, F. E. Hudson, S. Freer, K. M. Itoh, D. N. Jamieson, and J. C. Mccallum, J. Phys. Condens. Matter 27, 154205 (2015).

[5] B. E. Kane, Nature (London) 393, 133 (1998).

[6] J. L. O’Brien, S. R. Schofield, M. Y. Simmons, R. G. Clark, A. S. Dzurak, N. J. Curson, B. E. Kane, N. S. McAlpine, M. E. Hawley, and G. W. Brown, Phys. Rev. B 64, 161401 (2001).

[7] A. M. Tyryshkin, S. A. Lyon, A. V. Astashkin, and A. M. Raitsimring, Phys. Rev. B 68, 193207 (2003).

[8] A. R. Stegner, C. Boehme, H. Huebl, M. Stutzmann, K. Lips, and M. S. Brandt, Nat. Phys. 2, 835 (2006).

[9] J. J. L. Morton, A. M. Tyryshkin, R. M. Brown, S. Shankar, B. W. Lovett, A. Ardavan, T. Schenkel, E. E. Haller, J. W. Ager, and S. A. Lyon, Nature (London) 455, 1085 (2008).

[10] S. Simmons, R. M. Brown, H. Riemann, N. V. Abrosimov, P. Becker, H.-J. Pohl, M. L. W. Thewalt, K. M. Itoh, and J. J. L. Morton, Nature (London) 470, 69 (2011).
[11] M. Fuechsle, J. A. Miwa, S. Mahapatra, H. Ryu, S. Lee, O. Warschkow, L. C. L. Hollenberg, G. Klimeck, and M. Y. Simmons, Nat. Nanotechnol. 7, 242 (2012).

[12] H. Büch, S. Mahapatra, R. Rahman, A. Morello, and M. Y. Simmons, Nat. Commun. 4, 2017 (2013).

[13] D. P. Franke, F. M. Hrubesch, M. Künzl, H.-W. Becker, K. M. Itoh, M. Stutzmann, F. Hoehne, L. Dreher, and M. S. Brandt, Phys. Rev. Lett. 115, 057601 (2015).

[14] D. P. Franke, M. P. D. Pflüger, P.-A. Mortemousque, K. M. Itoh, and M. S. Brandt, Phys. Rev. B 93, 161303 (2016).

[15] G. Wolfowicz, M. Urdampilleta, M. L. W. Thewalt, H. Riemann, N. V. Abrosimov, P. Becker, H.-J. Pohl, and J. J. L. Morton, Phys. Rev. Lett. 113, 157601 (2014).

[16] M. Singh, J. L. Pacheco, D. Perry, E. Garratt, G. T. Eyck, N. C. Bishop, J. R. Wendt, R. P. Manginell, J. Dominguez, and T. Pluym, Appl. Phys. Lett. 108, 062101 (2016).

[17] G. W. Morley, M. Warner, A. M. Stoneham, P. T. Greenland, J. V. Tol, C. W. M. Kay, and G. Aeppli, Nat. Mater. 9, 725 (2010).

[18] P. A. Mortemousque, S. Berger, T. Sekiguchi, C. Culan, R. G. Elliman, and K. M. Itoh, Phys. Rev. B 89, 155202 (2014).

[19] K. Saeedi, M. Szech, P. Dluhy, J. Salvail, K. Morse, H. Riemann, N. Abrosimov, N. Nötzel, K. Litvinenko, B. Murdin, and M. Thewalt, Sci. Rep. 5, 10493 (2015).

[20] G. Wolfowicz, A. M. Tyryshkin, R. E. George, H. Riemann, N. V. Abrosimov, P. Becker, H.-J. Pohl, M. L. W. Thewalt, S. A. Lyon, and J. J. L. Morton, Nat. Nanotechnol. 8, 881 (2013).

[21] A. Morello, J. J. Pla, F. A. Zwanenburg, K. W. Chan, K. Y. Tan, H. Huebl, M. Möttönen, C. D. Nugroho, C. Yang, J. A. van Donkelaar, A. D. Alves, D. N. Jamieson, C. C. Escott, L. C. L. Hollenberg, R. G. Clark, and A. S. Dzurak, Nature (London) 467, 687 (2010).

[22] J. J. Pla, K. Y. Tan, J. P. Dehollain, W. H. Lim, J. J. L. Morton, D. N. Jamieson, A. S. Dzurak, and A. Morello, Nature (London) 489, 541 (2012).

[23] A. Bienfait, J. J. Pla, Y. Kubo, X. Zhou, M. Stern, C. C. Lo, C. D. Weis, T. Schenkel, D. Vion, D. Esteve, J. Morton, and P. Bertet, Nature (London) 531, 74 (2016).

[24] C. Eichler, A. J. Sigillito, S. A. Lyon, and J. R. Petta, Phys. Rev. Lett. 118, 037701 (2017).

[25] C. W. Zollitsch, K. Mueller, D. P. Franke, S. T. B. Goennenwein, M. S. Brandt, R. Gross, and H. Huebl, Appl. Phys. Lett. 107, 142105 (2015).

[26] S. J. Angus, A. J. Ferguson, A. S. Dzurak, and R. G. Clark, Nano Lett. 7, 2051 (2007).

[27] A. Bruno, G. D. Lange, S. Asaad, K. L. V. D. Enden, N. K. Langford, and L. Dicarlo, Appl. Phys. Lett. 106, 182601 (2015).

[28] K. G. Lyon, G. L. Salinger, C. A. Swenson, and G. K. White, J. Appl. Phys. 48, 865 (1977).

[29] C. A. Swenson, J. Phys. Chem. Ref. Data 12, 179 (1983).

[30] R. B. Roberts, J. Phys. D 15, L119 (1982).

[31] NIST Standard Reference Material 739, Certificate (NIST, Gaithersburg, MD, 1991).

[32] F. C. Nix and D. Macnair, Phys. Rev. 60, 597 (1941).

[33] A. Morello, C. C. Escott, H. Huebl, L. H. Willems van Beveren, L. C. L. Hollenberg, D. N. Jamieson, A. S. Dzurak, and R. G. Clark, Phys. Rev. B 80, 081307 (2009). 
[34] J. J. Pla, A. Bienfait, G. Pica, J. Mansir, F. A. Mohiyaddin, Z. Zeng, Y. M. Niquet, A. Morello, T. Schenkel, J. J. L. Morton, and P. Bertet, Phys. Rev. Appl. 9, 044014 (2018).

[35] J. Bardeen and W. Shockley, Phys. Rev. 80, 72 (1950).

[36] C. Herring and E. Vogt, Phys. Rev. 105, 1933 (1957).

[37] T. Thorbeck and N. M. Zimmerman, AIP Adv. 5, 087107 (2015).

[38] M. Veldhorst, C. H. Yang, J. C. C. Hwang, W. Huang, J. P. Dehollain, J. T. Muhonen, S. Simmons, A. Laucht, F. E. Hudson, and K. M. Itoh, Nature (London) 526, 410 (2015).

[39] D. K. Wilson and G. Feher, Phys. Rev. 124, 1068 (1961).

[40] H. Huebl, A. R. Stegner, M. Stutzmann, M. S. Brandt, G. Vogg, F. Bensch, E. Rauls, and U. Gerstmann, Phys. Rev. Lett. 97, 166402 (2006).

[41] L. Dreher, T. A. Hilker, A. Brandlmaier, S. T. B. Goennenwein, H. Huebl, M. Stutzmann, and M. S. Brandt, Phys. Rev. Lett. 106, 037601 (2011).

[42] D. Pines, J. Bardeen, and C. P. Slichter, Phys. Rev. 106, 489 (1957).

[43] A. Schweiger and G. Jeschke, Principles of Pulse Electron Paramagnetic Resonance (Oxford University Press, New York, 2005).

[44] See Supplemental Material at http://link.aps.org/ supplemental/10.1103/PhysRevLett.120.167701, which includes Refs. [45-64], for further details on experimental methods, tight-binding modeling, DFT calculations, modeling the $g$ factor anisotropy, and complete data sets.

[45] Y. M. Niquet, D. Rideau, C. Tavernier, H. Jaouen, and X. Blase, Phys. Rev. B 79, 245201 (2009).

[46] M. Usman, R. Rahman, J. Salfi, J. Bocquel, B. Voisin, S. Rogge, G. Klimeck, and L. L. C. Hollenberg, J. Phys. Condens. Matter 27, 154207 (2015).

[47] B. Roche, E. Dupont-Ferrier, B. Voisin, M. Cobian, X. Jehl, R. Wacquez, M. Vinet, Y.-M. Niquet, and M. Sanquer, Phys. Rev. Lett. 108, 206812 (2012).

[48] H. Nara and A. Morita, J. Phys. Soc. Jpn. 21, 1852 (1966).

[49] J. Bernholc and S. T. Pantelides, Phys. Rev. B 15, 4935 (1977).

[50] W. E. Krag, W. H. Kleiner, and H. J. Zieger, in Proceedings of the 10th International Conference on the Physics of Semiconductors (Cambridge, Massachusetts, 1970), edited by S. P. Keller, J. C. Hensel, and F. Stern (USAEC Division of Technical Information, Washington, DC, 1970), p. 271.

[51] A. K. Ramdas and S. Rodriguez, Rep. Prog. Phys. 44, 1297 (1981).

[52] R. K. Zhukavin, K. A. Kovalevsky, V. V. Tsyplenkov, V. N. Shastin, S. G. Pavlov, H.-W. Hübers, H. Riemann, N. V. Abrosimov, and A. K. Ramdas, Appl. Phys. Lett. 99, 171108 (2011).

[53] D. K. Wilson and G. Feher, Phys. Rev. 124, 1068 (1961).

[54] N. Lipari, A. Baldereschi, and M. Thewalt, Solid State Commun. 33, 277 (1980).

[55] M. Diarra, Y.-M. Niquet, C. Delerue, and G. Allan, Phys. Rev. B 75, 045301 (2007).

[56] M. G. Holland and W. Paul, Phys. Rev. 128, 30 (1962).

[57] G. A. Samara and C. E. Barnes, Phys. Rev. B 35, 7575 (1987).

[58] J. P. Perdew, K. Burke, and M. Ernzerhof, Phys. Rev. Lett. 77, 3865 (1996).

[59] P. E. Blöchl, Phys. Rev. B 50, 17953 (1994).

[60] G. Kresse and J. Furthmüller, Phys. Rev. B 54, 11169 (1996).

[61] P. E. Blöchl, Phys. Rev. B 62, 6158 (2000).

[62] Y. M. Niquet, L. Genovese, C. Delerue, and T. Deutsch, Phys. Rev. B 81, 161301 (2010).

[63] P. Y. Yu and M. Cardona, Fundamentals of Semiconductors: Physics and Materials Properties (Springer, New York, 1999).

[64] R. Rahman, S. H. Park, T. B. Boykin, G. Klimeck, S. Rogge, and L. C. L. Hollenberg, Phys. Rev. B 80, 155301 (2009).

[65] L. Zhang, R. Barrett, P. Cloetens, C. Detlefs, and M. S. D. Rio, J. Synchrotron Radiat. 21, 507 (2014).

[66] P. Ross, Ph.D. thesis, University College London, 2017.

[67] E. L. Hahn, Phys. Rev. 80, 580 (1950).

[68] W. Kohn and J. M. Luttinger, Phys. Rev. 98, 915 (1955).

[69] J. M. Luttinger and W. Kohn, Phys. Rev. 97, 869 (1955).

[70] S. T. Pantelides and C. T. Sah, Phys. Rev. B 10, 621 (1974).

[71] G. Pica, G. Wolfowicz, M. Urdampilleta, M. L. W. Thewalt, H. Riemann, N. V. Abrosimov, P. Becker, H.-J. Pohl, J. J. L. Morton, R. N. Bhatt, S. A. Lyon, and B. W. Lovett, Phys. Rev. B 90, 195204 (2014).

[72] G. Pica, B. W. Lovett, R. N. Bhatt, and S. A. Lyon, Phys. Rev. B 89, 235306 (2014). 